\title{
Pre Donation Deferral a Single Centre Experience
}

\author{
Rahul Vasudev ${ }^{\star}$, Supreet Kaur, Abhishek Kumar, Balraj Singh, Namrata Paul, Upamanyu Rampal and Raina TR \\ New York Medical College, St Joseph's Regional Medical Center, 703 Main Street Paterson NJ, USA \\ *Corresponding author: Rahul Vasudev, New York Medical College, St Joseph's Regional Medical Center, 703 Main Street Paterson NJ, USA, Tel: +1 973 4134387 ; E- \\ mail: drrahulvasudev@gmail.com
}

Received date: Apr 27, 2016, Accepted date: May 25, 2016, Publication date: May 30, 2016

Copyright: () 2016 Vasudev R, et al. This is an open-access article distributed under the terms of the Creative Commons Attribution License, which permits unrestricted use, distribution, and reproduction in any medium, provided the original author and source are credited.

\begin{abstract}
It is well known that quite a large number of apparently healthy donors are not able to donate blood successfully because of varied reasons. These donors are deferred temporarily or permanently. The aim of the study was to analyze the Incidence of Blood Donor deferral and its various causes and use this information as a guide for promoting Voluntary Blood donation. All the Blood donors reporting at our Blood Bank in last 6 months period were analyzed retrospectively. Data of all the donors deferred due to various causes was analyzed at our Transfusion Medicine Centre. There were 7253 donors, of which 524 donors were deferred $(7.22 \%)$ for various reasons. Of the 7253 donors registered for donation, females constituted only $8.6 \%$. And deferral rate was about six times more for female $(29.05 \%)$ compared to male $(5.18 \%)$. The three most common reasons for deferral low hemoglobin levels, chronic diseases and medication. More studies should be done at state and national level so that enough data can be collected and national policies formulated and western parameters for deferral should not be followed as regional differences cause unnecessary deferrals of already limited donors. Moreover analysis of donor deferral pattern will help blood banks to formulate more focused donor screening approach.
\end{abstract}

Keywords: Blood donor; Donor deferral; Temporary deferral; Permanent deferral

\section{Introduction}

Our Center is an 850 bed tertiary care hospital with $100 \%$ bed occupancy throughout year. This study was done at Blood bank GMC Jammu one of the two Blood Banks under P.G Department of Immunohematology and Transfusion Medicine, GMC Jammu, which is a Regional Blood Transfusion Centre (RBTC) and largest blood collection centre in the region. The annual blood collection at our Blood Bank is $16000-18000$ units. The blood donor suitability is based on informed medical opinion and regulatory rules set by Director General Health Services [1]. Deferred donors are those donors who do not qualify the guidelines for allogenic blood donation. Deferral may be temporary or permanent depending on the reason. Selection of blood donors is a critical step in ensuring safety of both recipient and donor. Moreover, deferring prospective donors often leaves them with negative feelings about themselves as well as the blood donation process [2]. The goal is to maintain a balance between acceptable quality of blood and desired quantity. The aim of the study was to analyze the incidence of blood donor deferral and its various causes and use this information as a guide for promoting voluntary blood donation.

\section{Subjects and Methods}

All the Blood donors reporting at our Blood Bank in last 6 months were analyzed retrospectively. Blood donors were screened by medical officer on duty based on brief medical history which included filling up of pre-existing donor questionnaire and brief examination with regard to blood pressure, temperature, pulse and weight of donor. Hemoglobin was estimated by Hemocue method. The blood donor deferral criteria were based upon guidelines issued by Director General
Health Services, India, and Departmental S.O.P's. Donors deferred were categorized on basis sex, age groups and whether deferral was temporary or permanent and the reason for deferral. Different ratios were calculated and significance limit was set at 0.05 and chi square test was used to find statistical significance.

\section{Results}

A total of 7253 donors were registered at out Transfusion Center during this period of 6 months. Out of 7253 donors presenting to us 524 were deferred. The incidence of deferral at our centre was $7.22 \%$. A total of 343 males were deferred out of 6630 who reported to us for blood donation (5.18\%) and 181 females were deferred out of 623 reporting for donation (29.05\%), 91.4\%(6630) of the donors were males and females constituted only $8.6 \%(623)$ of the donors. The difference between males and females turning up for blood donation was highly significant ( $\mathrm{p}$ value $=0.000005$ ).

Age and sex distribution of deferred donors is given in Table 1. The difference between deferred donors in these age groups was statistically not significant.

Most of the of the donor deferral were due to temporary causes $(n=343,66.6 \%)$, permanent deferral of donors was done in $33.3 \%$ donors $(n=175)$. In our study the most common cause for temporary deferral was anemia in both in male and female donors. The next common causes of temporary deferral were medications for various reasons, $\mathrm{H} / \mathrm{O}$ tattooing, ear piercing, dental extraction, Donation less than 3 months, low body weight, Surgical causes and Alcohol intoxication. A very few number of cases were also deferred because of failed phelebotomy.

Permanent deferral of donors was done in $33.3 \%$ donors $(n=175)$. History of chronic diseases like hypertension with heart disease, diabetes mellitus on Insulin, epilepsy, thyroid and renal diseases were most common cause of permanent deferral. Other causes were 
Page 2 of 3

jaundice with suspected hepatitis $\mathrm{B}$ and $\mathrm{C}$ infection, high risk sexual behaviour. Six professional donors who had come to blood bank after receiving money from attendants of patients were also permanently deferred.

\begin{tabular}{|c|c|c|c|}
\hline Reason for deferral & Males & Females & Total \\
\hline Anemia & 76 & 110 & $186(53.2 \%)$ \\
\hline Medication & 37 & 6 & $43(12.3 \%)$ \\
\hline $\mathrm{H} / \mathrm{O}$ tattooing, ear piercing, dental extraction & 32 & 2 & $34(9.7 \%)$ \\
\hline Donation less than 3 months & 31 & 2 & $33(9.4 \%)$ \\
\hline Under weight & 13 & 10 & $23(6.5 \%)$ \\
\hline Surgical causes & 8 & 3 & $11(3.1 \%)$ \\
\hline Alcohol intoxication & 10 & 0 & $10(2.8 \%)$ \\
\hline Pregenancy/lactation & 0 & 2 & $2(0.57 \%)$ \\
\hline Failed phelebotomy & 2 & 5 & $7(2 \%)$ \\
\hline Total & 209 & 140 & 349 \\
\hline
\end{tabular}

Table 1: Showing causes of temporary deferral.

\section{Discussion}

The deferral rate at our center was $7.22 \%$ which is lower than most of the studies (8-15\%) as reported by Chaudhry [3], Agnihotri [4], Ranveet [5] and higher than found by Sundar et al. (5.84\%) [6]. This may be due to the huge demand and supply gap that we face and pressures us to lax our donor deferral criteria so as adequate supply can be maintained. Deferral of donor has a negative feeling about self and adverse effect on limited donor pool available. A balance must be maintained between available safe blood and adequate quantity.

Majority of the donors i.e., $91.6 \%$ in our study were males and female donors were only $8.4 \%$, Chaudary et al. [3] and Arslan [7] found similar differences in male and female donation trends which may be due to hesitation in society towards female donation. There is still huge difference between number of males and females turning up for donation. Number of males turning up for donation is nine times more. The chances of female getting deferred are six times more as compared to males. Education and motivation of female donor pool may help to decrease this disparity and improve our donor pool.

Most of the deferrals (66.6\%) were due to temporary causes such as anemia, medication, tattooing, previous donation less than three months ago as compared to permanent deferral causing 33.3\% Custer et al. reported $68.5 \%$ temporary and $31.5 \%$ permanent deferral [8]. Such temporarily deferred donors can be motivated to return back after proper education about deferral criteria. Providing literature in donor counselling regarding various deferral causes may help donors to self-defer themselves and come for donation when they are ready. Temporary donors are donors are donors that have been deferred due to causes like anemia medication and can return for donation after specific period depending on the reason for temporary deferral. Whereas permanently differed donors due to causes like HIV hep B,C cannot return for donation.

Most common cause of deferral overall was anemia (35.4\%) which was also the most common cause of deferral in males $(22.1 \%)$ and females $(60.7 \%)$. In a study by Halperin et al. the three most common short term deferral were low hemoglobin level, colds and/or sore throats, and elevated temperature [9], whereas that by Ranveet et al. under-weight, under-age, and low haemoglobin levels [5]. This is due to high prevalence of anemia in our community especially in females some of the unpublished studies done in this region of country showing prevalence of anemia as high as $80 \%$ in rural female population. Donors deferred due to anemia should be educated and treated for anemia making this group of deferred donor fit will have huge impact on deferral rates and decrease them. The hemoglobin criteria should be according to the reference range obtained from population of the region, western reference ranges should not applied to local population.

Chronic diseases like severe uncontrolled diabetes, hypertension, cardiac disease, asthma, epilepsy, autoimmune disease etc. caused $26.9 \%$ deferral this is due to high incidence on diabetes and hypertension in our country. Hypertension can lead to deferral of a significant percentage of prospective blood donors as evident in our study and another by Bahadur et al. [10]. However, any blood donor suffering from a marked degree of hypertension has to be bled with care as in such cases the sudden removal of 350 or $450 \mathrm{ml}$ of blood may precipitate a cerebral catastrophe [11]. This could be tragic for the donor as well as blood center bleeding such donor. Patients with hypertension and diabetes should not be out rightly rejected but assessed carefully as reduction of deferral in this group can cause large decrease in deferral rates.

Medication like antibiotics, vaccination, alcohol intake, tattooing ear piercing, donation less than 3 months etc. also cause significant number of deferrals, education of donors about these can help donors to self-defer themselves. Donors deferred due to these can also be decreased by educating the donors about these deferral criteria's and providing IEC material.

Failed phlebotomy caused only $1.3 \%$ of the deferrals. Deferred relatives could feel need to hire professional donor further endangering the safety of blood number of professional donors presenting to us were 6 who were permanently deferred, these professional donors are at high risk of HIV, Hepatitis and other diseases. 
Citation: Vasudev R, Kaur S, Kumar A, Singh B, Paul N, et al. (2016) Pre Donation Deferral a Single Centre Experience. J Blood Disord Transfus

In USA blood center approximately $83 \%$ of blood donors successfully donate, but $13 \%$ are rejected because of donor suitability issue. One percent is rejected for the positive test, which is often nonspecific or false positive and $2 \%$ to $4 \%$ of the phlebotomies are not successful [11].

Regions like ours are accident prone and terrorist related casualties are a regular feature, in such time of need we do not have a stable voluntary donor pool to fall back upon. Loss of donor after deferral has adverse effect on limited donor availability and there is need to monitor and asses the necessity and effectiveness of such deferrals. Temporary deferral should be actively and aggressively managed so that this donor pool is not lost permanently and after treatment, education and motivation they return back to the donor pool. More studies should be done at state and national level so that enough data can be collected and national policies formulated and western parameters for deferral should not be followed as regional differences cause unnecessary deferrals of already limited donors. Moreover analysis of donor deferral pattern will help blood banks to formulate more focused donor screening approach.

\section{References}

1. Newman B (2001) Blood donor suitability and allogeneic whole blood donation. Transfus Med Rev 15: 234-244.
2. Brecher ME (2005) AABB Technical Manual. (15thedn.) Bethedsa: AABB press, p: 101

3. Chaudhary RK, Gupta D, Gupta RK (1995) Analysis of donor-deferral pattern in a voluntary blood donor population. Transfus Med 5: 209-212.

4. Agnihotri N (2010) Whole blood donor deferral analysis at a center in Western India. Asian J Transfus Sci 4: 116-122.

5. Kaur P, Basu S, Marwaha N (2002) A reappraisal of underrlysing causes in donor deferral. Ann Natl Acad Med Sci 38: 93-99.

6. Sundar P, Sangeetha SK, Seema DM, Marimuthu P, Shivanna N (2010) Pre-donation deferral of blood donors in South Indian set-up: An analysis. Asian J Transfus Sci 4: 112-115.

7. Arslan O (2007) Whole blood donor deferral rate and characteristics of the Turkish population. Transfus Med 17: 379-383.

8. Custer B, Johnson ES, Sullivan SD, Hazlet TK, Ramsey SD, et al. (2004) Quantifying losses to the donated blood supply due to donor deferral and miscollection. Transfusion 44: 1417-1426.

9. Halperin D, Baetens J, Newman B (1998) The effect of short-term, temporary deferral on future blood donation. Transfusion 38: 181-183.

10. Bahadur S, Jain S, Goel RK, Pahuja S, Jain M (2009) Analysis of blood donor deferral characteristics in Delhi, India. Southeast Asian J Trop Med Public Health 40: 1087-1091.

11. Newman BH (2004) Whole-blood donation: blood donor suitability and adverse events. Curr Hematol Rep 3: 437-443. 